\title{
La Internacionalización e Interculturalidad como Ejes transversales de la Responsabilidad Social Universitaria en una Experiencia Educativa Virtual
}

Hákim Krayem, Mónica Rubiette

Universidad Veracruzana, México rhakim@uv.mx

Canal Martínez, Margarita Edith

Universidad Veracruzana, México

mcanal@uv.mx

\author{
Del Callejo Canal, Diana Donají \\ Universidad Veracruzana, México \\ ddelcallejo@uv.mx \\ Fortuno Hernández, Josefa Carolina \\ Universidad Veracruzana, México \\ jfortuno@uv.mx
}

Resumen - Se destaca la intervención de la innovación pedagógica para transversalizar la Internacionalización e Interculturalidad, como ejes de responsabilidad social universitaria en la Experiencia Educativa (EE) Virtual Ética Contemporánea. El objetivo fue determinar su importancia en la formación estudiantil. Se implementaron estrategias innovadoras planeadas y organizadas de la propuesta Guía del Docente de la Universidad Veracruzana. Los resultados mostraron que la mitad de los estudiantes consideraron la Interculturalidad e Internacionalización en situaciones donde la Ética se aplica.

Palabras clave - Innovación pedagógica; Planeación por competencias; Estrategias innovadoras; Ética; Interculturalidad; Internacionalización;

Abstract - The intervention of pedagogical innovation is highlighted to mainstream Internationalization and Interculturality, as axes of university social responsibility in the virtual Educational Experience (EE) Contemporary Ethics. The objective was to determine its importance in student training. Planned and organized innovative strategies of the proposed Teacher's Guide of the

Interconectando Saberes, 2020

ISSN: 2448-8704
Universidad Veracruzana were implemented. The results showed that half of the students considered Interculturality and Internationalization in situations where Ethics was applied.

Keywords - Pedagogical innovation; Planning by competences; Innovative strategies; Ethics; Interculturality; Internationalization;

\section{INTRODUCCIÓN}

La Asociación Nacional de Universidades e Instituciones de Educación Superior Educación (ANUIES), considera a la educación como un bien social cuya influencia en la formación integral de las personas, el desarrollo y bienestar de las naciones es incuestionable; por ello, impele para que las instituciones de educación superior estratégicamente deban transformarse, innovarse, replantear sus fundamentos y finalidades, como un reto y compromiso de formar universitarios que sean portadores de un nuevo impulso humano

Fecha de Recepción: 06 de febrero de 2020

Fecha de Aceptación: 12 de mayo de 2020

Fecha de Publicación: 24 de julio de 2020 
y social para responder con pertinencia, equidad, eficacia y eficiencia a una sociedad a la que se deben (Asociación Nacional de Universidades e Instituciones de Educación Superior, 2012).

Por lo anterior, la Universidad Veracruzana (UV) desde el marco de su Programa Estratégico 2017 2021, denominado Pertenencia y Pertinencia, incluyó tanto en el cuerpo del documento como en la descripción de los Ejes Rectorales, estrategias, programas y líneas de acción que enfatizan la Internacionalización y la Interculturalidad pertinencia de las acciones innovadoras docentes con las políticas universitarias-, como políticas y acciones a integrarse de forma transversal dentro de las funciones sustantivas de la institución, tales como: Docencia, Investigación y Difusión y Extensión.

Así mismo, al implementar estrategias innovadoras, la UV responde a lo que se sugirió en la Conferencia de la UNESCO (Organización de las Naciones Unidas para la Educación, la Ciencia y la Cultura, 2009), a saber:

La Educación Superior debería asumir el liderazgo social en materia de creación de conocimientos de alcance mundial para abordar retos mundiales, entre los que figuran la seguridad alimentaria, el cambio climático, la gestión del agua, el diálogo intercultural, las energías renovables y la salud pública. (p. 5)
Bajo este tenor de ideas, la UV, no sólo se sumó a este reto y compromiso internacional, sino que adoptó la Responsabilidad Social Universitaria (RSU) como una política institucional para asegurar que su gestión alcance el objetivo de formar ciudadanos integrales, superando el enfoque de proyección social y extensión universitaria como "apuestas" de buena intención sobre su función central de formación integral, producción de conocimientos e interacción social (Vallaeys, 2007), y así poder asumir la verdadera exigencia de la RSU. Si bien la RSU está en proceso de construcción, la UV, la define como:

[...] el compromiso de la Universidad para difundir y poner en práctica una serie de conocimientos y valores en la formación profesional, en los procesos de investigación, gestión y extensión. Estas funciones deben estar enfocadas a las soluciones de problemáticas sociales mediante cuatro procesos: gestión ética y sustentable de la Universidad, formación de ciudadanos conscientes y comprometidos, generación y difusión de conocimientos socialmente pertinentes, $y$ participación social para un desarrollo más equitativo y sostenible (Ladrón de Guevara, 2019). 
Derivado de lo anterior, asumimos también en nuestra función docente y como facilitadores el compromiso con la responsabilidad social; dicho compromiso se centra en guiar nuestras acciones desde la filosofía del actual Modelo Educativo Integral (MEI) de la UV, el cual concibe la necesidad de realizar innovaciones planeadas, organizadas y concretas para que mediante la práctica docente y en su aplicación con los estudiantes, se logre el desarrollo de las competencias a través de aprendizajes significativos y esto permita la generación de nuevas formas de ser-hacer, así como de convivencia en sociedad.

En este contexto, la propuesta de innovar y transversalizar algunos de los ejes del proyecto institucional de Responsabilidad Social Universitaria (RSU) de la UV, denominado: Internacionalización e Interculturalidad, constituye un esfuerzo para hacer operativos los principios de su modelo educativo y los procesos de enseñanzaaprendizaje que ocurren a través de las Experiencias Educativas (EE) de los diferentes programas educativos, prestando atención a cómo se interrelacionan los saberes teóricos, heurísticos y axiológicos, para lograr que los estudiantes desarrollen sus propios desempeños y competencias de manera autorregulada, descubran los distintos enfoques que se posicionan dentro de su área de conocimiento, partiendo de una planeación didáctica y metodológica para el cumplimiento de las actividades de aprendizaje.
Para responder a este proyecto institucional universitario, se centró la atención en elaborar e implementar una estrategia didáctica de intervención sustentada en un enfoque constructivista en la EE denominada Ética Contemporánea, perteneciente al Área de Formación de Elección Libre (AFEL) ofrecida en modalidad virtual a través de la plataforma EMINUS, durante el periodo agosto 2018 - enero 2019, con el objetivo de que ésta impactara, transformara y condujera a la innovación del proceso educativo, potenciando las capacidades de los estudiantes, vinculándolos con una realidad exterior desde la mirada nacional, internacional e intercultural, respetando la riqueza de las experiencias locales y culturales -visualización de distintos enfoques de una situación real-, mediante la realización de análisis críticos, creativos y propositivos que les permitiera concebir ideas $y / 0$ explicaciones para transformar e innovar en un contexto determinado en busca del bien común.

En esta intervención pedagógica para transversalizar la Internacionalización e Interculturalidad dentro de la EE de Ética Contemporánea, se partió desde uno de los procesos considerados más complejos de un modelo por competencias: ${ }^{1}$ la Evaluación del desempeño, puesto que en las evidencias de aprendizaje que los estudiantes construyen, se deben manifestar los logros alcanzados, en el caso que nos ocupa, la comprensión de situaciones

\footnotetext{
${ }^{1}$ Guía para el diseño de proyectos curriculares, con el enfoque
} de competencias. Universidad Veracruzana. 
donde se aplica la Ética según los enfoques nacionales, internacionales e interculturales.

De allí la importancia de que los facilitadores en modalidades virtuales, conciban su labor como una responsabilidad y un desafío ético para el desarrollo social, interviniendo para apoyar el desarrollo de las competencias de los estudiantes; es decir, innovando desde la comunicación las estrategias didácticas, los recursos y métodos de enseñanza, elaborando materiales, etcétera; convirtiéndose así en un acompañante en el proceso de aprendizaje, pero que además, incida y trascienda el ámbito de la formación de manera auténtica en los cambios que nos benefician a todos: la formación de ciudadanos responsables en su desempeño profesional, con saberes pertinentes que hayan recuperado la riqueza de las experiencias locales y globales, que participen activamente en la solución de problemas, que preserven la cultura y que consoliden los valores de la convivencia, atributos sustanciales de la RSU.

El presente artículo se integra de dos partes, en el apartado de Desarrollo, se expone el contexto de la EE para comprender la complejidad de los saberes que los estudiantes van a desarrollar con la orientación del facilitador, así como las situaciones a las que se van a enfrentar para vivenciar su aprendizaje, generar y autoorganizar sus conocimientos, y con ello favorecer el ejercicio de la Ética en los ámbitos nacionales, internacionales e interculturales.
De igual manera y en este mismo apartado, se presentan brevemente las estrategias metodológicas utilizadas con el propósito de integrar los conocimientos, habilidades y actitudes aprendidas de manera significativa durante el curso, para recuperar saberes de otras EE cursadas y para promover procesos en los cuales el alumno se cuestione estas concepciones, las contraste con hechos, con vivencias o emociones y esto le permita adquirir otras nuevas mediante un progresivo cambio conceptual y actitudinal, promoviendo su crecimiento personal crítico y autónomo (Yus, 1998). De igual modo, se presentan otras estrategias como la presentación/investigación de estudios de caso de una realidad concreta. Todo lo anterior, para lograr que los estudiantes generen un pensamiento complejo para ubicar toda la información que necesitan conocer y utilizar en un contexto real y en conjunto (Morin, 2003).

En el segundo apartado, se presentan los resultados de las estrategias didácticas implementadas, así como las conclusiones y algunas propuestas de mejora que incitan y motivan para continuar en la búsqueda de mecanismos que promuevan el aprendizaje de los estudiantes, basados en su propia intervención y construcción del conocimiento.

Por último, es pertinente mencionar que este proyecto de intervención pedagógica responde a las orientaciones de dos cursos tomados pertenecientes al proyecto institucional de la UV en torno a la RSU: Formación universitaria integral: 
Transversa e Internacionalización del Curriculum, ya que en las actividades realizadas se construyeron planeaciones específicas de la EE que impartimos, entrelazando los contenidos con los ejes transversales de RSU para propiciar la reflexión de los estudiantes con sus conocimientos disciplinares, habilidades y actitudes en la toma de decisiones desde distintos enfoques $y$ perspectivas: regional, estatal, nacional, internacional e intercultural, e identificar en qué medida su actuar es congruente con lo que se espera del mundo contemporáneo.

\section{DESARROLLO}

\section{Contexto de la EE}

Con la intención de contextualizar la EE de Ética Contemporánea, se presentan algunas características, previo a describir el desarrollo, tipo de intervención y metodología aplicada.

- La EE de Ética Contemporánea, pertenece al $\mathrm{AFEL}^{2}$ y está dirigida a la formación complementaria del desarrollo integral de los estudiantes, considerada en el plan de estudios de todos los programas educativos de la UV. Esta EE se gestiona académica y administrativamente desde el Sistema de Enseñanza Abierta, por tratarse de una modalidad virtual. Esta área, diversifica el contacto con ambientes de trabajo con visiones multi e interdisciplinarias, promueve resultados y procesos innovadores que enriquecen la opción profesional en la que se están formando los estudiantes, ofreciéndole alternativas de saberes y experiencias de aprendizaje.

- La Unidad de Competencia de la EE de Ética Contemporánea consiste en lo siguiente: que el estudiante analice hechos, opiniones, posturas, teorías y casos reales en situaciones individuales, sociales, científicas y profesionales, fortaleciendo su pensamiento ético y sus habilidades para la toma de una postura y decisión frente a escenarios que impliquen el discernimiento ético y moral, con la finalidad de ser un individuo íntegro que conviva de manera armónica y congruente con su entorno individual y colectivo. Lo anterior en un ambiente de respeto y tolerancia (Programa de la EE, 2018, p. 2).

- Para lograr el cumplimiento de esta Unidad de Competencia en un espacio de aprendizaje virtual, en donde la tecnología ha permeado a la escuela y se ha adentrado a la vida de las aulas, como facilitadores hemos tenido que modificar nuestro rol y no sólo por el uso de herramientas tecnológicas per se, sino que ha venido a transformar las concepciones y prácticas pedagógicas comunes (Parra, 2012), provocando que la planeación y diseño de estrategias de enseñanza-aprendizaje impulsen a los estudiantes a ser autónomos en su aprender a aprender. Se requiere de una alta motivación por parte de los

\footnotetext{
2 El AFEL, "es un espacio de expresión y crecimiento personal que brinda a los estudiantes la posibilidad de ampliar oros saberes y experiencias de aprendizaje, distintos 0 complementarios a la disciplina que estudian- y con esto
}

contribuir a la consolidación de ciudadanos responsables" (Universidad Veracruzana - Sistema de Enseñanza Abierta, 2020). 
estudiantes tanto para leer indicaciones, seguirlas y tener la disciplina de cumplir en tiempo y forma con las actividades; requiere también, de un soporte técnico en tiempo real por parte de quienes administran las plataformas tecnológicas y por último y no menos importante, las habilidades necesarias como la planeación y comunicación estratégica implementada por el facilitador para lograr oportunidades de interacción constante, para mantener el interés de los estudiantes a través de un seguimiento puntual, constante y permanente a través de mensajes y retroalimentaciones, por mencionar algunos.

- Las características del grupo donde se aplicó la intervención pedagógica estuvo integrado por 35 alumnos de diferentes regiones universitarias, áreas académicas y programas educativos diversos. Con una participación del $69 \%$ de mujeres y $31 \%$ por hombres. Dicha diversidad de estudiantes permitió que tanto ellos, como el facilitador, amplíen su perspectiva sobre el tema a tratar, toda vez que las participaciones, permiten conocer distintos enfoques $y$ perspectivas desde su formación profesional, contribuyendo las reflexiones generadas mediante análisis críticos sobre los temas y logrando la Unidad de

3 En el estudio de casos "el estudiante aprende a fundamentar y tomar decisiones, reconstruye y analiza situaciones reales en forma individual y/o grupal; revisa documentos, los ordena, propone alternativas de solución y decide al respecto" (s.f., p. 6). Recuperado de: https://www.uv.mx/desarrollocurricular/disenocurricular/
Competencia, objetivo de la EE y que incide en el desarrollo de los saberes teóricos, heurísticos y axiológicos.

Bajo este contexto, se procedió a la construcción de la propuesta didáctica en tres etapas, con base en el momento en que se utilizan las estrategias constructivistas: antes de la instrucción, durante y después de la instrucción (Díaz Barriga y Hernández, 2002).

\section{Estrategias metodológicas}

Para el logro del objetivo de esta intervención pedagógica constructivista y dar cuenta de las acciones innovadoras desarrolladas, considerando que la instrucción fuera estructurada y fácilmente aprovechada por los estudiantes (organización en espiral), de acuerdo con las experiencias y contextos, se implementaron las siguientes estrategias de aprendizaje:

- Estudio de Casos $^{3}$ y la Investigación, ${ }^{4}$ a través de la presentación y/o búsqueda o indagación de problemáticas, hechos actuales y reales que permitiera a los estudiantes reconocerlos, analizarlos, exponer sus propias ideas sobre los temas, discernir de manera compleja y holista sobre un tema en particular, dando paso a nuevas concepciones y pensamientos de la sociedad que los rodea desde el punto de

\footnotetext{
${ }^{4}$ En la Investigación "el estudiante aprende a identificar problemas, planear el proceso de investigación y comunica los resultados encontrados a través del desarrollo de un proyecto (s.f., p. 10). Recuperado de: https://www.uv.mx/desarrollocurricular/disenocurricular/
} 
vista ético ${ }^{5}$ y al mismo tiempo, les permitiera configurar ideas complejas sobre lo que debiera ser el vivir con ética y utilizar los conocimientos aprendidos de su disciplina o profesión con actitud de respeto y ayuda al otro y además, a través del uso de diferentes recursos digitales fortalecimiento y desarrollo de saberes teóricos, heurísticos y axiológicos en su aprender construyendo-, es decir, pasando de un planteamiento "temático" a un planteamiento "problemático" (Yus, 1998).

- Aunado a lo anterior y a la metodología empleada, se entretejieron algunas actividades alineadas a los contenidos, actividades de aprendizaje y objetivos de desempeño ya establecidos en el programa de la EE, es decir, se analizaron casos desde el enfoque y perspectiva de la Internacionalización y la Interculturalidad conocimiento en construcción-, con el fin de fortalecer en los estudiantes su pensamiento y habilidades para la toma de una postura y decisión frente a distintos escenarios, enfatizando que el conocimiento del entorno nunca se agota en sí mismo, puesto que, cuanto más se conoce la realidad, mayores interrogantes se nos abren y más expectativas se nos van creando (Yus, 1998). Estas actividades aportaron elementos para una convivencia responsable socialmente y armónica en lo individual y colectivo.

\section{Inicio y final del curso}

Sin duda, el facilitador es uno de los actores primordiales en el proceso de aprendizaje: marca el tono y el tipo de relaciones que surgen en el curso, debido al lugar de autoridad que le confiere la institución y los estudiantes, y por ser el principal organizador de la dinámica grupal (Pérez, 2009), por ello, al inicio del curso, a través del Foro de Bienvenida, se establecen los mecanismos de comunicación permanentes y se solicita a los estudiantes compartan con el grupo cierta información: Programa Educativo y periodo que cursan, región, sus pasatiempos, subir una foto formal a la plataforma para identificarlos y acercarse en una realidad virtual y responder los siguientes cuestionamientos:

Al inicio: para obtener un diagnóstico sobre los conocimientos y experiencia de los estudiantes en torno al contenido temático de la $\mathrm{EE}$, se les pregunta sobre:

1.- Expectativas para elegir cursar la EE.

2.- ¿Cómo consideran a la Ética y su aplicación en el mundo contemporáneo?

3.- Si es o no la primera vez que cursan una $\mathrm{EE}$ en modalidad virtual.

Al final: para apoyar al facilitador no sólo a la asignación de una calificación, sino para identificar los logros de desempeño, siendo este cuestionario un criterio más de evaluación, se determina sobre:

\footnotetext{
${ }^{5}$ Se considera a la Ética en términos generales como el estudio de los valores que guían el comportamiento de los seres humanos.
} 
1.- ¿Qué sé sobre la Ética? (saberes teóricos).

2.- ¿Qué habilidades desarrollé durante el curso? (saberes heurísticos).

3.- ¿Qué valores éticos he comprendido o recordado? (saberes axiológicos).

4.- ¿Desde tu disciplina, cómo puedes aplicar la ética desde diferentes contextos: locales, nacionales, internacionales, interculturales? (RSU).

\section{Implementación de estrategias de Internacionalización e Interculturalidad}

Las actividades de aprendizaje implementadas como son las de investigación y análisis crítico relacionado con la Internacionalización y la Interculturalidad, desde la perspectiva y contenido de la Ética, tuvieron la finalidad de contribuir a la adquisición de competencias internacionales e interculturales en los estudiantes. Por mencionar algunos ejemplos, se planearon estrategias como la revisión de los Derechos Humanos, poniendo énfasis en los derechos particulares de los indígenas, se analizaron estudios de caso y Legislación que se desarrollan en otros países en el área del Cuidado y Preservación de la Salud casos como la Eutanasia y Homicidio asistido-, se redactaron conclusiones en inglés y los estudiantes entregaron un Proyecto integrador final, en donde retomaron los conocimientos aprendidos, desde una mirada ética, compleja, holista y crítica, pudieron crear y construir un trabajo integrador ético-social, demostrando las competencias desarrolladas durante el curso.
Para tener mayor claridad en las actividades realizadas, algunas se describen a continuación:

- Actividad EC2. "Ética y Valores". Se invita a la reflexión sobre las características implícitas de los valores qué motivan al individuo a tomar decisiones y que éstas se traducen en comportamientos y actitudes. Para ello revisan y analizan un video sobre el caso de una periodista en la guerra en Oriente y leen un material de apoyo a fin de que cuenten con elementos teóricos para su participación en el Foro de discusión o debate, bajo los lineamientos previamente establecidos y elaborados por el facilitador. Esta acción permite a los estudiantes establecer relaciones razonables $y$ significativas con el mundo.

- Actividad EC5. "Casos y Cosas". Análisis crítico de los principales conceptos relacionados con el tema de la Salud como un derecho universal de todo ser humano. Los estudiantes eligen algún reporte, película o documental disponibles en la plataforma, investigan información complementaria, se plantean preguntas en torno a qué harías si estuvieras frente a una situación similar y responden cuestionamientos de análisis en un formato predeterminado. Así mismo, se investigan casos recientes en el mundo en donde se identifiquen violaciones (conductas antiéticas) al Derecho a la Salud y cómo éstos fueron resueltos. 
- Actividad EC9. "Cambiando nosotros para cambiar a México". Trabajo colaborativo. Los estudiantes leen para comprender los principales conceptos relacionados con la Corrupción y estrategias anti-corrupción. Posteriormente a través de mensajes y otras formas de comunicación que ellos deciden utilizar, se contactan con su equipo de trabajo y toman acuerdos para elaborar una Infografía sobre el tema; de manera ilustrativa y creativa muestran su postura e ideas que como sociedad podemos realizar para combatir la Corrupción, especialmente en la población indígena o las zonas más vulnerable del país y proponen soluciones. Cabe mencionar que este trabajo es socializado de forma individual en sus redes sociales y envían evidencias de desempeño de dicha socialización en la plataforma.

- Actividad EC11. Esta actividad pretende hacer una revisión de los Derechos Humanos como compromisos, derechos y obligaciones que guían nuestro vivir en lo personal y profesional y de que su aplicación depende de la Ética; se pone especial énfasis en los Derechos Humanos de los indígenas y lo investigado se plasma en una Revista de Divulgación.

- Actividad EC14. Consiste en la consolidación de los contenidos y aprendizajes que integran 3 requisitos fundamentales: la investigación, la complejidad y el empleo de las Tecnologías de la Comunicación (TIC). En este proyecto integrador final, los estudiantes eligen un tema de los revisados y seleccionan una opción de evidencia: Cuento, cortometraje, historieta, caricatura periodística, etc. Cada opción cuenta con una ficha técnica que tienen que construir e incluye una reflexión personal y conclusiones, misma que se les solicita sea en inglés. Además de responder un cuestionario final.

\section{Estrategias de Comunicación}

La comunicación o presencia del facilitador en un ambiente virtual se considera vital, toda vez que brinda seguridad a los estudiantes de que "alguien" está caminando con ellos en su aprendizaje y desarrollo de competencias. El adecuado desarrollo de la comunicación y las interacciones genera un clima socioafectivo propicio para el proceso de aprendizaje (Visser, 2002; García, 2002 en Pérez, 2009).

Por lo anterior, en cada inicio de actividad se envía un mensaje con las indicaciones paso a paso sobre las acciones a realizar, agregando significados 0 ejemplos a palabras o siglas consideradas poco cotidianas; las retroalimentaciones a los ensayos, foros, infografías, proyecto final, etcétera se realizan los primeros días hábiles de la entrega de la evidencia de aprendizaje, a fin de que los estudiantes conozcan sus aciertos, debilidades 0 puntos ausentes en tiempo y forma y consideren las observaciones para mejorar su propio aprendizaje; al concluir el $50 \%$ de las actividades del curso, el facilitador envía un mensaje a los estudiantes en riesgo escolar, a fin de exhortarlos y motivarlos para el logro de la acreditación de la EE, identificar alguna problemática y recordándoles que, en el 
programa AFEL, no existen exámenes extraordinarios ni otras modalidades de acreditación, tal y como lo marca el Estatuto de los Alumnos de la UV.

Por último, en cada Retroalimentación Individual, se reconoce y felicita al estudiante por cumplir con todos los lineamientos solicitados; se exhorta a mejorar a los que casi cumplen con la totalidad y se exhorta a quienes no lo logran aún.

\section{Resultados}

Del total de los 35 estudiantes inscritos, 22 de ellos fueron promovidos satisfactoriamente (62.86\%), 5 estudiantes no lograron la promoción (14.29\%) y 8 estudiantes (22.85\%) se inscribieron, pero no entregaron ninguna de las 14 actividades por lo que se consideran estudiantes de Baja de la EE. Lo anterior refleja, sin duda, que la metodología aplicada rindió buenos resultados y que como toda planeación académica es factible de mejorarse y de implementar nuevas estrategias para la mejora continua del proceso educativo.

En cuanto a las actividades en donde se llevaron a cabo estrategias de transversalización para comprender la Internacionalización e Interculturalidad, se puede afirmar que sólo el 50\% del total de los estudiantes consideraron estos elementos en sus trabajos, lo cual interpretamos que ellos, casi en su mayoría, no leen las indicaciones que por la vía de mensajes se enviaron y sólo enfocaron la atención en las indicaciones ya establecidas en EMINUS. Sin embargo, las evidencias entregadas bajo estos criterios fueron excelentes, cumpliendo con las competencias esperadas, esto es, los objetivos y evidencias de desempeño para dichas actividades implicaron en los estudiantes investigar, discernir, reflexionar y mostrar una postura en el análisis de hechos y problemáticas reales.

A manera de ejemplo, se muestran algunas de las evidencias de desempeño de los estudiantes:

- Estudios de caso e investigación sobre la Ética aplicada al ámbito de la Salud/ Internacionalización:

En el caso de la Organización Mundial de la Salud (OMS), tanto la práctica de Eutanasia pasiva y doble efecto son permitidas, pero para la Asociación Médica Mundial por sus siglas en inglés (WMA) infiere lo siguiente:

- La eutanasia, es decir, el acto deliberado de poner fin a la vida de un paciente, aunque sea por voluntad propia o a petición de sus familiares, es contraria a la ética. Sin embargo, esto no impide al médico respetar el deseo del paciente de dejar que el proceso natural de la muerte siga su curso en la fase terminal de su enfermedad (WMA, 2013).

- Por lo anterior se reafirma que la eutanasia entra en conflicto con los principios éticos básicos de la práctica médica, por lo que insta a todas las asociaciones médicas nacionales y los médicos a no 
participar en la eutanasia, incluso si está permitida por la legislación nacional 0 despenalizada bajo ciertas condiciones.

- Sin embargo, en el 2002, Holanda se convirtió en el primer país en legalizar la eutanasia y el suicidio asistido. Las condiciones son que el paciente tenga una enfermedad incurable, o un dolor insoportable, así como la exigencia en plena conciencia del paciente.

- Posteriormente se legalizó en Bélgica, Suiza y Luxemburgo, así como en el estado de California, Oregón y Washington, Vermont y recientemente en Hawái. (Evidencia de respuesta de un estudiante).

- Para el caso de la transversalización de la Interculturalidad, en la Figura 1 se muestra un ejemplo (evidencia de respuesta de un estudiante) para identificar los Derechos Humanos plasmados en una Revista de Difusión donde se enfatizan los derechos más comprometidos de los pueblos indígenas.
Figura 1. Ejemplo de portada del contenido utilizado por un estudiante sobre el tema de Interculturalidad.

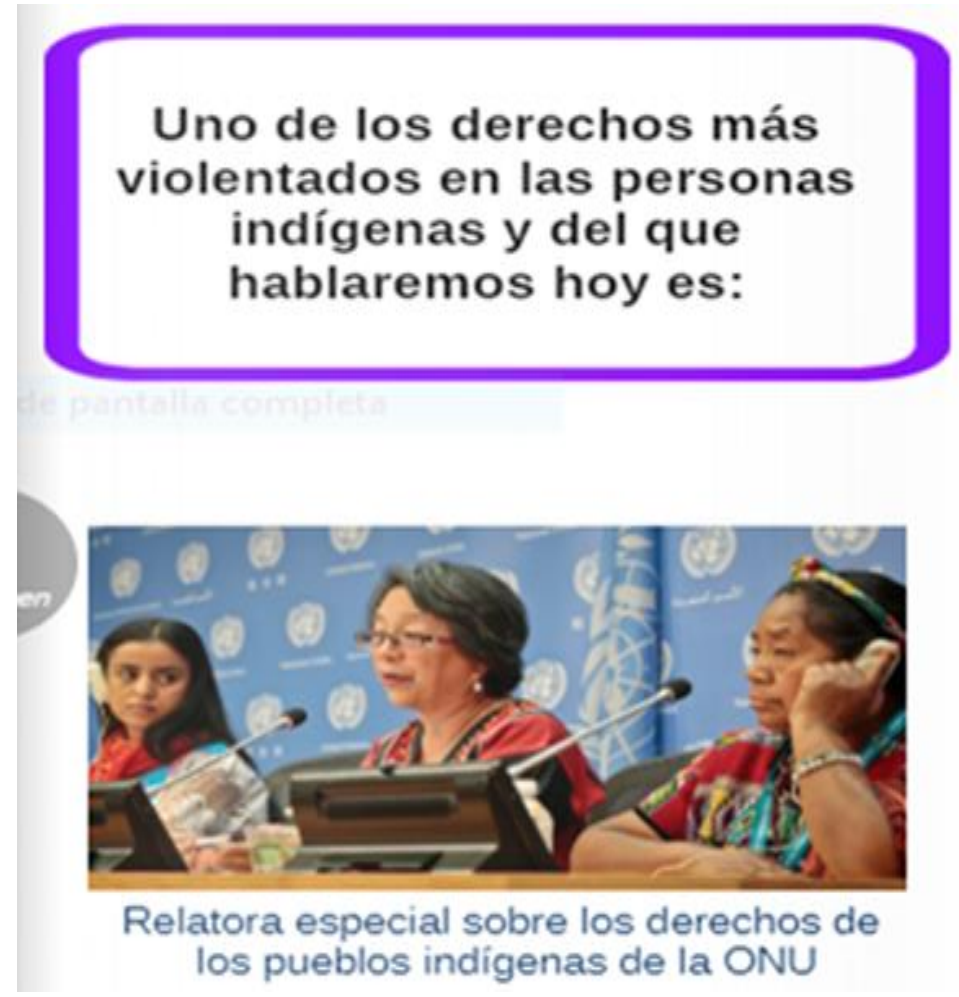

Fuente: Evidencia de desempeño de un estudiante sobre los Derechos Humanos e Interculturalidad.

Otro punto que resaltar es que al inicio del curso se les preguntó a los estudiantes sobre su entendimiento sobre qué es la Ética y su aplicación; las palabras para definirla fueron:

- Saber lo que es Bueno y malo.

- Es lo Moral y los valores.

- Respeto.

- Comportamientos adecuados.

- Criterio.

Al final del curso, en el proyecto integrador, se les solicitó una conclusión y reflexión sobre lo que significaba la Ética y su aplicación desde la disciplina que están estudiando; los resultados fueron gratificantes, por ejemplo: 
1. ¿Qué sé sobre la Ética? (saberes teóricos): La definición de Ética, qué son los valores, sobre la deshonestidad académica, el poder para cambiar a México, la divulgación de nuestros derechos a las personas que debemos defender, saber sobre hechos que suceden en poblaciones vulnerables y cuáles son sus derechos. Que la Ética interviene en muchas áreas y disciplinas. Códigos de ética profesionales y de las empresas.

2. ¿Qué habilidades desarrollé durante el curso? (saberes heurísticos): Desarrollé distintas habilidades durante el curso, como son Involucramiento de TIC, Soluciones innovadoras a problemas reales, habilidad para cuestionarme y responder, evitar el estrés y visión global.

3. ¿Qué valores éticos he comprendido o recordado? (saberes axiológicos): Los valores éticos que reforcé en esta EE fueron respeto, libertad, justicia, honestidad y tolerancia, seguridad en uno mismo, empatía.

4. Aplicación de la ética en la vida personal y profesional con compromiso social: En cada experiencia educativa que cursé pude notar el cambio en mi manera de pensar día con día, abriendo más mi panorama de manera personal y profesional. En un futuro tomare en cuenta todo lo aprendido en esta EE para el mejoramiento y crecimiento profesional y vivir armónicamente con los otros y poder hacer comparaciones de cómo se toman decisiones en otros lugares o países (Evidencia de respuesta de un estudiante).
Los resultados de promoción y logro de competencias de la EE de Ética Contemporánea, con suficiencia, pertinencia y congruencia, nos indica que si bien un alto porcentaje de estudiantes acreditaron la EE, desarrollando la Unidad de Competencia, reconocemos que el estudio de la Ética, la Interculturalidad, la Internacionalización -y de otros contenidos disciplinares y transversales-, específicamente en modalidades virtuales, tiene aún mucho por realizar en función del nuevo rol del facilitador para estructurar ambientes de aprendizaje que promuevan en los estudiantes el aprender a conocer, aprender a hacer, aprender a convivir y aprender a ser (Delors, 1996).

Por ello, considerando esta experiencia y resultados, afirmamos que como facilitadores, comprometidos socialmente desde nuestro quehacer, debemos diseñar, planear e implementar estrategias complejas, como son la reflexión y análisis de contenidos, comprensión lectora, creatividad e imaginación, investigación, redacción de ensayos, uso de herramientas digitales, respeto y tolerancia a la opiniones de otros y todo ello, vinculando lo aprendido con una realidad concreta, desde ámbitos y perspectivas locales y globales, que permitan en los estudiantes el desarrollo de competencias internacionales e interculturales para insertarse al mundo contemporáneo.

Se afirma que resulta imprescindible que los discursos curriculares rompan las fronteras, si es que deseamos coadyuvar al modelo por competencias establecido en nuestra universidad y considerar lo que, en la Conferencia de la 
Organización de las Naciones Unidas para la Educación, la Ciencia y la Cultura, (Organización de las Naciones Unidas para la Educación, la Ciencia y la Cultura, 2009) se concluyó:

La Educación Superior debe no sólo proporcionar competencias sólidas para el mundo de hoy y de mañana, sino contribuir además a la formación de ciudadanos dotados de principios éticos, comprometidos con la construcción de la paz, la defensa de los derechos humanos y los valores de la democracia (Comunicado de la Conferencia, Organización de las Naciones Unidas para la Educación, la Ciencia y la Cultura 2009, p. 2).

\section{REFERENCIAS}

Asociación Nacional de Universidades e Instituciones de Educación Superior (ANUIES) (2012). Evaluación, certificación y acreditación en la educación Superior en México. Colección Documentos. México.

Compilación de estrategias metodológicas de aprendizaje para el diseño de Experiencias Educativas (S/F): https://www.uv.mx/desarrollocurricular/disen o-curricular/

Delors, J. (1996). Los cuatro pilares de la educación, en La educación encierra un tesoro. Informe a la UNESCO de la Comisión internacional sobre la Educación para el siglo XXI. Madrid, España: Santillana/UNESCO. pp. 91-103.
Díaz Barriga F. y Hernández (2002) Estrategias docentes para un aprendizaje significativo. Una interpretación constructivista. México: Mc Graw Hill. Recuperado de: http://www.educaweb.com/noticia/2008/12/0 1/alfabetizacion-digital-docentes-3349/

Grof, Stanislav (2000). La revolución de la conciencia. Barcelona: Kairós.

Guía para el diseño de proyectos curriculares, con el enfoque de competencias. Universidad Veracruzana (2005). México. Recuperado de: https://www.uv.mx/dgdaie/guia-diseno/

Ladrón de Guevara, Sara D. (2019). Responsabilidad Social Universitaria. Universo. Sistema de noticias de la UV. Recuperado de: https://www.uv.mx/prensa/banner/uv-afirmocompromiso-para-impulsar-politica-deresponsabilidad-social-universitaria/

Morin, Edgar (2003). Introducción al pensamiento complejo. Barcelona: Gedisa.

Organización de las Naciones Unidas para la Educación, la Ciencia y la Cultura (UNESCO) (2009). Education transforms lives. Recuperado de: http://www.unesco.org/education/WCHE200 9/comunicado es.pdf

Parra, C. (2012). TIC, conocimiento, educación y competencias tecnológicas en la formación de maestros. Nómadas, 36, 145-159.

Pérez A. María del Socorro (2009). La comunicación y la interacción en contextos virtuales de aprendizaje. Revista de Innovación Educativa. Vol. 1. Universidad de Guadalajara. Recuperado de:

http://www.udgvirtual.udg.mx/apertura/index. php/apertura/article/view/15/18

Programa de la Experiencia Educativa Ética Contemporánea (2018). Recuperado de: https://www.uv.mx/dgdaie/files/2018/12/etica MEIFversion-avalada-SEA-240806.pdf 
Universidad Veracruzana - Sistema de

Enseñanza Abierta (SEA) (2020).

Experiencias Educativas Virtuales AFEL-

SEA. Recuperado de:

https://www.uv.mx/sea/eevirtuales/

Universidad Veracruzana (2017). Programa

Estratégico 2017-2021, Pertenencia y

pertinencia. Recuperado de:

https://www.uv.mx/documentos/files/2019/05

/pte-2017-2021.pdf

Vallaeys, François. (2007) La responsabilidad social universitaria: un nuevo modelo universitario contra la mercantilización. Revista Iberoamericana de Educación Superior, Vol. V, núm. 12, 2014, pp. 105117. Instituto de Investigaciones sobre la Universidad y la Educación. México.

Recuperado de:

https://www.redalyc.org/pdf/2991/299129977 006.pdf

Yus Ramos, Rafael (1998). Síntesis: un modelo didáctico para la transversalidad, en Temas transversales, hacia una nueva escuela. Barcelona: Graó. Recuperado de: http://www.uv.mx/dgdaie/files/2012/11/CPP_DC-Yus-Sintesis-un-modelo-didactico.pdf 